\title{
Exploration of Galactic Structures beyond the Sun toward the anti-center of the Milky Way
}

\author{
Yan $\mathrm{Xu}^{1}$ and Heidi Newberg ${ }^{2}$ et al. \\ ${ }^{1}$ National Astronomical Observatories, Chinese Academy of Sciences, Beijing China \\ email: xuyan.jade@gmail.com \\ ${ }^{2}$ Rensselaer Polytechnic Institute, USA \\ email: newbeh@rpi.edu
}

\begin{abstract}
We map the stellar distribution on Hess diagram in the Anti-Center roughly in the boxes $130<l<230,-30<b<-10$ and $10<b<30$. There are 'extra components' associated with the anti-center structures of figure 1 of Newberg et al. (2002). The turnoff point of the structure in the North sky is at $16^{m} .5$ and the turnoff point in the South is at $17^{m} .5$. In our work, these structures can be found in all of the longitude in our box that can't be explained by standard thin or thick disk models. The distance of the North structure is about $2 \mathrm{kpc}$ (we call it the North near structure) and the galactic height is about $0.7 \mathrm{kpc}$, the distance of the South structure is about $4-6 \mathrm{kpc}$ (we call it the South middle structure). The Vgsr distribution of stars selected along the North near structure has a kinematic distribution similar to that of thick disk stars. But the metallicities of these stars are quite similar to the metallicity distribution of thin disk stars. We try to explain these structures with wave structure of the Galactic plane.
\end{abstract}

Keywords. substructure, anti-center of the Galactic disk

\section{Discussion}

1: If the extinction can produce the asymmetry of star counts between north and south sky?

The north near structure is about $1^{m}$ brighter than south middle structure. Besides, extinction direction is almost parallel with main sequence direction, extinction can't cause such kind of pattern in the Hess diagram.

2: If the structures are spiral arm? The Perseus spiral arm and Outer arm have similar distance with north near structure and south middle structure. However these structures are above and below the the plane about 1Kpc. If the Galactic height is too high for spiral arm?

3: If the structures are tidal streams? The north near structure has thin disk like metallicity. Usually the tidal streams are metal poor.

4: If the structures are warp or flare? The disk is 'warped' and 'flared'. But the north near structure is strong and only appear around $2 \mathrm{Kpc}$.

5: They are more like result of resonance with the bar or wave structure of the disk itself.

\section{Reference}

Newberg, H., Yanny, B., Rockosi, C. et al. 2002, APJ, 569, 249 